\title{
Proceeding
}

\section{Gaze and performance of an elite karate exponent: a case study.}

\author{
EDIN KARDIN SUWARGANDA \\ National Sports Institute of Malaysia, Kuala Lumpur, Malaysia,
}

\begin{abstract}
Kardin, E. (2015). Gaze and Performance of an Elite Karate Exponent: a Case Study. J. Hum. Sport Exerc., 9(Proc2), pp.S672-S676. The choice of attacks by Karate exponents is not well understood but is of importance to coaching practices. This case study explores gaze tracking sequences prior to attacking and seeks possible associative actions performed by the karateka. One Malaysian Karate exponent with over 8 years experience in the National team volunteered to participate in a simulation match of 2 minutes while wearing the Mobile Eye glasses (ASL, USA). Gaze was recorded at $25 \mathrm{~Hz}$ and was synchronised with an additional camera $(25 \mathrm{~Hz})$ recording the performance from just outside the mat. Gaze fixations were manually coded along 6 different areas (head, above the head, chest, abdominal, arm and leg) of the opponent. Also performance tactics (attack or counter) and actions (lunges, kick, punch and blocks) by the participant were manually notated. Gaze fixation sequences were selected from the start of an action back to when fixation had stabilized after the last action. The sequences were then clustered using the longest common sequence method in the TraMineR package of R-project statistical software. The clusters, similar in fixations sequence and length, were then associated with the tactics and actions performed. In total 23 fixation sequences and a same number of actions were identified. The most frequent and longest fixations were concentrated around the body and head of the opponent. The dendrogram showed three major types of clusters. Type 1 was mostly associated with counter attacks (79\%) that included punches (57\%). The second and third clusters contained the same number of attacks as counters and were mostly associated with lunges (50\%) and kicks (38\%). Furthermore looking at the lengths of the sequences within each cluster, type 2 and 3 had longer lengths than type 1 . Therefore, these two clusters could essentially be considered as one despite differences in eye fixation sequence. The counter chudan (punch to the body) technique was used the most (7 times) and is associated with cluster type 1. Attacking kicks ( 5 times) and backward lunges ( 5 times) on the other hand are associated with the combination of type 2 and 3 . However, it is rather the length of the fixation sequence in between the attacks than the area of fixations themselves that was found to be associated with the actions by this karateka. Short fixation sequences evoke a fast punching response. This is likely due to pressured situations where the distance to the opponent was reduced quickly by an attacking opponent. While longer fixation sequences are followed by (evading) lunges and kicks to disrupt the opponent and keep distance from the opponent without a direct attempt to score. Key words: KARATE, STUDY, MOBILE EYE, GAZE, ATTACKS.
\end{abstract}

\footnotetext{
Corresponding author. Jalan Barat,Bukit Jalil,57000 Kuala Lumpur,Wilayah Persekutuan Kuala Lumpur, Malasia E-mail: edin@isn.gov.my

Asia Pacific Conference on Performance Analysis of Sport, 21-24 April 2014. Langkawi, Malaysia.

JOURNAL OF HUMAN SPORT \& EXERCISE ISSN 1988-5202

(c) Faculty of Education. University of Alicante

doi:10.14198/jhse.2015.10.Proc2.05
} 


\section{INTRODUCTION}

In karate many types of attacks are at the disposal of the karateka to attempt a score. Other than the variety of punches and kicks, e.g. straight, reverse, hooked, chopped, and executed either from front side or back side. There are also take downs, sweeps and of course some combination of these techniques. Furthermore techniques are used in the context of attack, defend or counter attack, which are precluded by a variety of different tactical movements prior to the technical executions. Lastly, karatekas can score points ranging from 1 to 3 when hitting the body or the head. So together this creates a large number of attacks the karateka can utilise.

At the National Sports Institute of Malaysia the performance analyses center is working closely together with the National Karate team on technical and tactical feedback. One of the major challenges identified with the team is for each karateka to select and flawlessly execute the most appropriate technique(s) under the circumstances and to do that at an optimum timing and distance. Even though, the training of the team is designed to influence this ability. The choice of attacks by Karate exponents during matches is not well understood. The 'information' Karatekas process through their vision plays a leading role in the decisions that they make.

Unfortunately, the scientific literature is void with regards to decision making in Karate. However, related to this topic Williams and Elliott (1999) did investigate visual search strategy and response (movement) under different anxiety conditions. They found superior anticipation by experts compared to novices. Similar results were found in research by Mori et.al. (2002), they made an investigation into the reaction times and anticipatory skills by expert and novices. But again, nothing is known about how about what karatekas perceive could possibly influence their tactical and technical choices.

To get better insight on how the gaze of a Karateka could influence the tactical and technical choices made, I set up a case study to explore gaze sequences prior to attacking and seek possible associative technical choices performed by the karateka.

\section{METHODS}

One Malaysian Karate exponent (male, 27 years old) with over 8 years experience in the National team volunteered to participate in a simulation match of 2 minutes while wearing the Mobile Eye glasses (ASL, USA). The Mobile Eye tracks the gaze of the Karateka. It has two cameras, one forward looking camera that captures the viewing field of the Karateka and the other camera focussed on an eye from which the pupil tracking occurs. Fixations during gaze were recorded at $25 \mathrm{~Hz}$ and were displayed as a red cross on the video of the forward looking camera (ASL Result software). Necessary calibration was done before and during the simulation match. An additional camera $(25 \mathrm{~Hz})$ recorded the technical performances from just outside the mat. The forward looking camera and the additional camera were synchronized by way of a flash light signal.

Fixations were manually coded along 6 different areas (head, above the head, chest, abdomen, arms and legs) of the opponent. From the additional camera, performance tactics (attack or counter) and technical actions (kicks, punches, lunges, blocks etc) by our subject were also manually notated in Excel (Microsoft Software). Fixation sequences were defined as from the start of an action back to when fixation had stabilized after the last action. So, each fixation sequence was determined as the period in between attacking actions in accordance with the performances as seen on the (additional) video. The sequences were then clustered using the longest common sequence method in the TraMineR package of R-project 
statistical software. The longest common sequence calculates 'distances' between sequences in the following manner. For a particular code (or element) in the sequence which is equal to the code of another sequence, the calculated distance will not be increased (or increased by zero). If however another code differs at the next place, the calculated distance will be increased by one (1). This has an advantage as the distance between sequences that for example differ in one code in the middle of a sequence would only be increased with one (1) even though the rest of the codes appear at a different place within the sequence. Finally, if a certain place and thus also a code is absent in the other sequence, the calculated distance will be increased by two (2). Thus the 'distance' increases with more non-similar codes in the fixations sequence and with longer differences in length between two sequences. Distances between all sequences were calculated and sequences with relative small differences were clustered. All fixation sequences were time dependent. Therefore, it was identified which fixation sequence was followed up with what tactical and technical actions. This made it possible to associate the clusters of fixation sequences with some tactics and actions performed.

\section{RESULTS}

In total 23 fixation sequences and a same number of actions were identified. Across all sequences the most frequent and longest fixations were concentrated around the body $(26.9 \%)$ and head $(30.3 \%)$ of the opponent. Most often, these fixations switched between body and head. Additionally, our Karate exponent was more interested in the arms (16.3\%) rather than the legs (5.5\%). Another $4.0 \%$ of the time was fixated somewhere above the head and $17 \%$ of the time was spend either blinking, in saccadic period (search gaze) or as unstable fixations (during technical executions).

The dendrogram (figure 1) showed three major types of clusters with the distance represented as height on the vertical axis. The type 1 or the first cluster was the biggest cluster containing 13 sequences. Type 2 or the second cluster was the smallest cluster consisting of only 3 sequences and type 3 or third cluster was build up out of 7 sequences. In figure 2 the sequences are depicted by cluster (or type) and colour coded with green (Arms), purple (Body), orange (Head), yellow (Leg) and blue (above head, S). Looking at the length of the coloured sequences it can be seen that the type 1 sequences are shorter in length than type 2 and 3. The colour coding also indicates that type 2 has more and longer fixations to the arm (green) compared to type 1 and 3. From the coding of tactical and technical actions it was calculated that type 1 was mostly associated with counter attacks (79\%) that could included punches (57\%). Furthermore looking at the lengths of the sequences within each cluster, type 2 and 3 had similar lengths and were different from type 1. Therefore, these two clusters could essentially be considered as one cluster despite differences in eye fixation sequence. Together, the second and third clusters contained the same number of attacks as counters and were mostly associated with lunges forward or lunges backward $(50 \%)$ and/or kicks (38\%). 


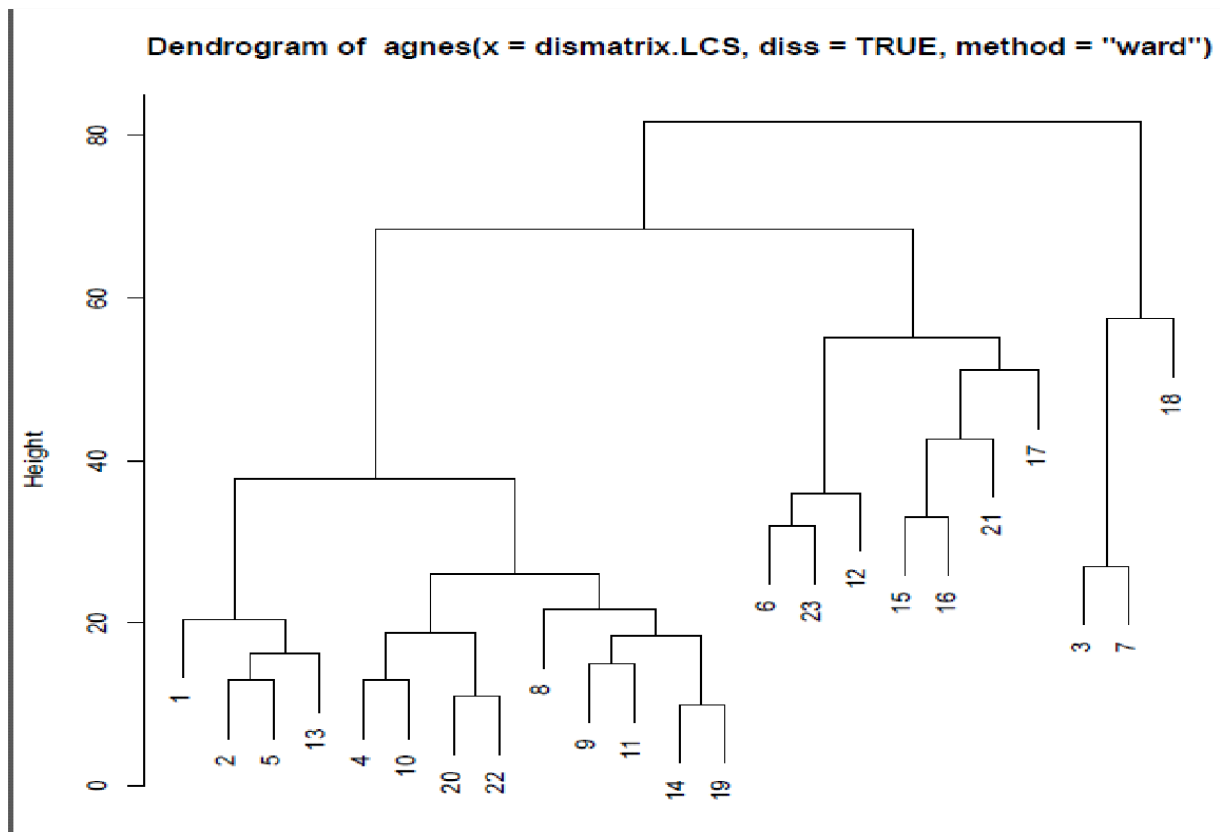

Figure 1. Dendrogram of fixation sequences number 1-23 clustered according to distance.

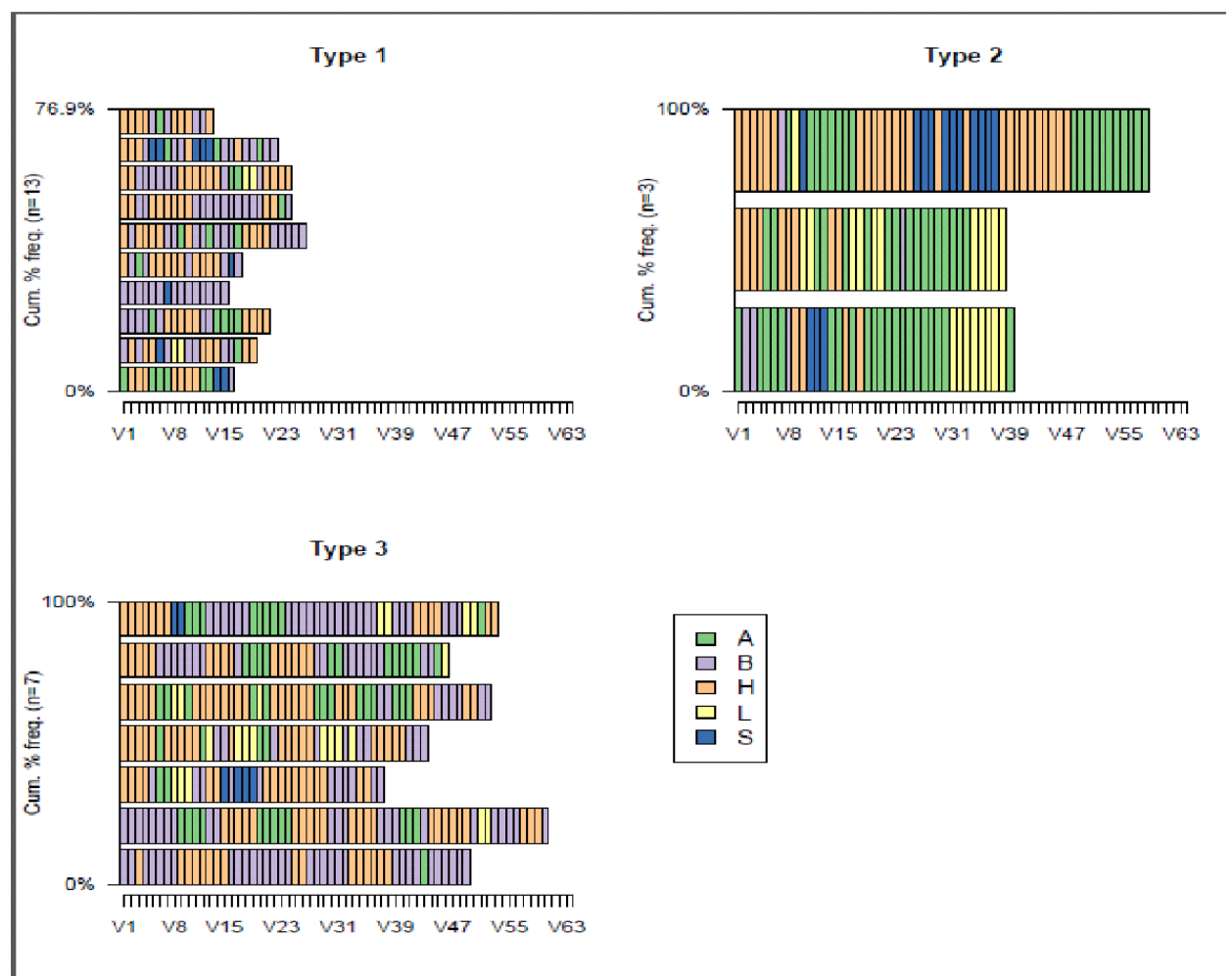

Figure 2. Three cluster type graphs with maximum 10 colour coded fixation sequences.

\section{DISCUSSION}

Even though three different clusters were identified from the sequential analyses in R-project, no clear difference could be seen looking at the colour codes within the sequences between clusters. Except, 
perhaps the type 2 cluster in which relatively more and longer fixations were on the arms of the opponent. However, there seem to be a difference in the tactics and techniques associated with the clusters. Specifically, between cluster 1 on one side and cluster 2 and 3 on the other side. But these differences could not obviously be associated with sequences of fixations themselves. Instead, the associations had to be dependent on something else. Looking more closely at differences between cluster 1 and the combination of cluster 2 and 3 together, we can see a clear difference in length of fixations. Cluster 1 contained the shorter fixation sequences, while cluster 2 and 3 contained the longer sequences. This was the rationale of combining cluster 2 and 3 , consequently treating them as a single cluster despite differences in the fixation sequences.

The punch to the body or counter chudan technique was used the most (7 times) and is associated with cluster type 1. This is likely due to the pressured situations where the distance to the opponent reduces fast by an attacking opponent or by attacking initiative at possibly an optimum distance and timing. The attacking kicks (5 times) and backward or forward lunges (5 times) on the other hand are associated with the combination of cluster 2 and 3 . The forward lunges were executed as either an attacking threat while the backward lunges were executed as a reaction to attacking or forward movement by the opponent. Interestingly, the attacking kicks were also executed as a threat by keeping the knee at hip height and sometimes extending multiple kick from that knee position while hopping forward on the supporting leg. These tactical movements and techniques effectively control the distance with the opponent. It is to disrupt the opponent's action and keep distance from the opponent without a direct attempt to score. Perhaps the Karateka prefers to await his opportunity to attempt a score at the optimum distance and timing. It could be said that the experienced level of this Karateka have conditioned him to react in these two manners. These manners allow the karateka to control the pace and rhythm of the match. In future, additional research should take into account these timing preferences, together with an in depth exploration of sub sequences and the actions by the opponent.

\section{CONCLUSIONS}

This case study showed that the choice of technique was not determined by the fixation frequency, duration or sequence pattern. Rather it was the length of the fixation sequence in between the attacks that was found to be associated with the actions by this karateka. Short fixation sequences evoke a fast punching response, while longer fixation sequences are followed by lunges and kicks.

\section{ACKNOWLEDGEMENTS}

This case study was not possible without the help of the colleagues from the centre for biomechanics, the National Karate team and Chris Button for borrowing his equipment.

\section{REFERENCES}

1. Mori, S., Ohtani, Y., \& Imanaka, K. (2002). Reaction times and anticipatory skills of karate athletes. Human Movement Sciences, 21, p.p. 213-230.

2. Williams, A. M., \& Elliott, D. (1999). Anxiety, expertise and visual search strategy in karate. Journal of Sport and Exercise Psychology, 21, p.p. 362-375. 